\title{
Una revisión teórica sobre la adopción del comercio electrónico
}

A theoretical review of the adoption of e-commerce

\author{
Ronaldo Domingo Chavez Vilcahuaman ${ }^{a}{ }^{(\mathbb{D} *}$, Monica Nicole Aldaba Murrieta ${ }^{b} \mathbb{D}$, Carlos \\ Eduardo Corrales Baldoceda ${ }^{\mathrm{C}}$ \\ a,b,c Universidad Peruana Unión, Lima, Perú
}

\section{Resumen}

El propósito de este trabajo es presentar un breve acercamiento teórico de la adopción del comercio electrónico. Este conceptualmente se desplazó de una noción de estrategia de rentabilidad para las empresas que brindan servicios y organizaciones comerciales. El modelo de Aceptación Tecnológica propone pronosticar un grupo u organización que adoptan de una nueva tecnología con la finalidad de predecir el futuro de su uso. Una definición concluyente considerada para el comercio electrónico es que se considera como un implemento necesario e instrumento que permite al consumidor realizar actividades de compraventa donde el alcance de disponibilidad de acceso es ilimitado que genera rentabilidad para la empresa a través de sus dimensiones: seguridad percibida entendido como control de seguridad que se brinda al consumidor; la lealtad del consumidor siendo las actitudes que toma según sus experiencias y la expectativa que tiene frente a la empresa y por último la satisfacción siendo las expectativas del cliente del servicio brindado.

Palabras clave: Comercio electronico; satisfacción; lealtad; seguridad percibida

\section{Abstract}

The purpose of this paper is to present a brief theoretical approach to the adoption of electronic commerce (e-commerce). This conceptually started from a notion of profitability strategy for companies that provide services and commercial organizations. The Technological Acceptance model proposes to forecast a group or organization that adopt a new technology in order to predict the future of its use. A conclusive definition considered for e-commerce is that it is considered as a necessary implement and instrument that allows the consumer to

\footnotetext{
* Autor de correspondencia:

Tel.: +051-930153245

E-mail: ronaldochavez@upeu.edu.pe/monicaaldaba@upeu.edu.pe DOI: https://doi.org/10.17162/riva.v7i1.1418

Recibido: 20/09/2020 Aceptado: 10/11/2020
} 
carry out buying and selling activities where the scope of access availability is unlimited, which generates profitability for the company through its dimensions: perceived security understood as a security control that is provided to the consumer, the loyalty of the consumer which are the attitudes that they take according to their experiences and the expectation that they have towards the company and lastly the satisfaction which are the expectations of the client of the service provided.

Keywords: Electronic commerce, e-commerce, satisfaction, loyalty, perceived security

\section{Introducción}

La adopción del comercio electrónico para algunas empresas se hace dificultoso implementar esta tecnología por la falta de áreas organizacionales o gerenciales según Romero \& Mauricio (2012), por ende, en los últimos años fue de estudio y de captación por distintas entidades comerciales y de servicios (Lee \& Turban, 2001). La adopción del comercio electrónico es un recurso estratégico de competitividad (Tsuja \& Nishimura, 2002), por lo tanto, al medirlo permite saber la confianza, seguridad percibida, la lealtad y la satisfacción del consumidor o cliente, generando información de la adaptabilidad de los clientes en la organización que se está ejecutando dicha tecnología y por ello también una base de mejora en el sistema que trae consigo la rentabilidad a las empresas (Herrero \& Rodríguez, 2001).

Este estudio como utilidad permite un desarrollo teórico breve sobre el comportamiento de la adopción del comercio electrónico que influye en las empresas para su supervivencia en el mercado. Trayendo consigo beneficios para la entidad y para el consumidor, por ende, la venta competitiva y los recursos para combatir con otros mercados (Robaina \& Alzola 2006).

Según Ballesteros, Tavera, \& Castaño (2015), en la revisión mencionan que los ajustes del modelo confirman que la confianza influye directamente en la intención del uso, lo cual Carola, Aderete, \& Motta (2013) señalan como factores que más inciden en las diferencias en los niveles de adopción del comercio electrónico son el tamaño de las empresas y su nivel de madurez en la implementación de tecnologías de información. También Rodríguez, Gutiérrez \& Herrero (2006) mencionan que la evidencia emperica obtenida pone de manifiesto la mayor capacidad del Modelo de Aceptación Tecnológica para predecir la aprobación de la compra electrónica. En otro estudio realizado por Aponte (2013) muestra la existencia de diferencias significativas en la confianza hacia el comercio electrónico entre los consumidores con mucha y poca seguridad.

Este trabajo tuvo como objetivo general realizar una revisión teórica sobre la adopción del comercio electrónico, a través del desarrollo de un modelo teórico y sus dimensiones. $A$ su vez abordar el concepto teórico sobre el origen del comercio electrónico, sus dimensiones 
y el concepto del modelo teórico. Se procedió a conseguir artículos sobre teoría de la variable en estudio, teniendo acceso a base de datos de información científica, como sciencedirect, redalyc, scielo, scopus, google scholar, dialnet, repositorios, springer, alicia concyetc. Asimismo, se utilizó motores de búsqueda y gestores de información para la citación y la consignación de las referencias como Mendeley.

\section{Desarrollo o Revisión}

\subsection{Origen del comercio electrónico}

La expresión comercio electrónico aparece asociada con la evolución de las actividades sencillas y es aquí donde su concepto se empieza a difundir en la comunidad académica y profesional, de allí se define mediante dos líneas explicativas, la primera explicación es la aparición de la tecnología y la segunda explicación es la adopción de esta.

Según Rios (2014), menciona que a lo largo del tiempo se vinieron haciendo innovaciones desde las tareas más sencillas, hasta que llego la revolución industrial donde se ve el apogeo de la electricidad trayendo consigo la aparición de aparatos electrónicos, lo cual Oropeza (2018), afines de los años noventa se consolido una interdependencia en la economía donde las organizaciones vieron una oportunidad de realizar actividades comerciales por ende se dio el proceso de la oferta y la demanda.

El internet con el comercio electrónico se desarrolló de formas similares en los años sesenta teniendo el comercio electrónico cuatro generaciones, en la primera se vio la importancia y el impacto, en la segunda vieron la oportunidad como estrategia, en la tercera se vio el marketing en la red y la implementación de bases de datos y por último en la cuarta se enfocaron en el cuidado del diseño y aspecto Torres \& Guerra (2005), entre ellos tenemos a Everett (1995) que menciona la teoría de las innovaciones como canales de tiempo.

\subsection{Modelo teórico de aceptación tecnológica (TAM)}

El modelo propuesto por Fred D. Davis es un método que sirve como un instrumento para pronosticar a un grupo u organización la adopción de una nueva tecnología, tiene como finalidad predecir el futuro del uso de la tecnología. Establece dos variables para este modelo: utilidad percibida y facilidad de uso percibida.

De acuerdo con Yong, Rivas, \& Chaparro (2010) este método está especialmente diseñado para predecir la aceptación de los sistemas de información por los usuarios en las organizaciones, determinando el rechazo o aceptación de una determinada tecnología de información (Bedregal, Tupacyupanqui, Cornejo, \& Flores, 2019) y a su vez también determina explicar el proceso de aceptación de la tecnología de la información a nivel individual (Ramirez 
\& Rondan, 2014). En realidad, Bedregal et al., (2019) dice que este modelo está relacionado con las convicciones, las actitudes, las intenciones y el comportamiento del usuario, para predecir la aceptación de una TI, sin embargo, el modelo de aceptación tecnológica en el enfoque del comercio electrónico es la dirección que tiene el usuario mediante su comportamiento, la voluntad que esta toma mediante su racionalidad ante una situación (Yong, 2004).

Así mismo, Davis (1989) menciona que el objetivo de este modelo es explicar la relación entre la aceptación y adopción de la tecnología y, posteriormente, la intención de usarla en la organización, por tanto, Romero, Alarcón, \& Gómez (2011) menciona que el individuo cree, que, usando un determinado sistema, este aumentará su rendimiento en sus labores. Además, Ajzen (1991) añaden que este modelo les ofrece un efecto directo y positivo entre las actitudes hacia el uso, la intención de uso y el uso que el individuo hace de la Web. Por tanto, Davis (1989) sugiere que la utilidad y la facilidad de uso son determinantes en la intención que tenga un individuo para usar un sistema.

Respecto al modelo, Salazar \& Ramirez (2014) establecen las consecuencias de los factores externos en cuanto a la utilidad y la facilidad de uso percibidas, para adelantar o predecir el uso de las TIC. A su vez, la utilidad percibida, es cuando el individuo cree que usando un determinado sistema propuesto aumente su productividad (Romero, 2011). De la misma manera, Yong (2010) añade que la facilidad del uso percibido hace referencia que el individuo cree que, mediante el saber del uso de cualquier sistema determinado por la organización, este estará libre de cualquier esfuerzo que realice (Yong, 2004). También Zolait (2016) añade que dicha explicación se basaba en el análisis de las creencias y de las actitudes de los sujetos, así como en sus oportunidades y recursos.

\subsection{Comercio electrónico}

El comercio electrónico se entiende como un implemento necesario e instrumento que permite al consumidor realizar actividades de compraventa donde el alcance de disponibilidad de acceso es ilimitado y, a las empresas, generar rentabilidad.

Según Tsuja \& Nishimura (2002), el comercio electrónico es un nuevo método para hacer negocios tanto en el ámbito nacional como internacional, por ende, hace que la forma de competir sea distinta a la que ya estamos acostumbramos, y que sus operaciones se vuelven más rápidas y eficaces sin que nos tome un gran esfuerzo.

Potter (2001) citado por Fransi, Ramón, \& Fuentes (2013) señalan que el comercio electrónico proporciona productos y servicios exclusivos, donde los competidores no tienen el 
alcance de ejercer en un prolongado tiempo y ver lo que la competencia ofrece, siendo esto una ventaja competitiva de crear y producir productos únicos en el mercado.

Por ende, el comercio electrónico se refiere al manejo de las tecnologías de información y comunicación que se da en el proceso de transacciones comerciales, para que esta pueda tener relaciones con otras organizaciones y estas con el cliente, con la finalidad de crear utilidad y valor para la empresa, (Jones, Alderete, \& Motta, 2014).

\subsubsection{Confianza del consumidor.}

La seguridad percibida en un enfoque al comercio electrónico se define que los propietarios o empresas deben tener un control de seguridad garantizando al cliente 0 consumidor al momento de incluir sus datos, transacciones u entre otras actividades al web que están en buen alcance de seguridad por parte de la empresa.

Aponte (2013), señala que la seguridad percibida del consumidor en el enfoque hacia el comercio electrónico, es necesario que los negocios virtuales ofrezcan altas medidas de seguridad con virtud a la interacción de las transacciones y los datos personales garantizando la protección de éstos procedimientos o actividades sobre cualquier ataque cibernético, no tan solo aumentará su confianza, sino que pretenderá recoger todo aquello que puede generar incertidumbre al cliente (Melián \& Padrón, 2005).

Por otro lado, se define la seguridad percibida como la impresión que tiene el usuario con respecto a su información personal o financiera durante las transacciones de datos que brinde estén a salvo (Myung, Chung, \& Choong 2011). Por ello Kalakota y Winston (1996) citado por Belanger, Hiller, \& Smith (2002), dice que una amenaza ocasionada por falta de seguridad se define como un problema para causar dificultades económicas a los datos o recursos de red en forma de destrucción, divulgación, modificación de datos, denegación de servicio y / o fraude, desperdicio, y abuso. Sin embargo, Belanger, Hiller, \& Smith (2002), define que la seguridad percibida, es la protección que contrarresta aquellas amenazas que pueden hacerse a través de la red y ataques de transacciones de datos, o mediante acceso no autorizado, esta definición debe adaptarse para ser aplicable al consumidor para reconocer que la información del consumidor tiene valor.

Malca (2001), recomienda que la seguridad es una tarea constante, que los propietarios de los sitios web deben tener mayor control de ello. La óptima medida de seguridad es no incluir material que no pueda darse solución o que pueda perderse completamente. El objetivo es que solo las personas autorizadas puedan alterar o reemplazar la información en el sitio web.

\subsubsection{Lealtad del Consumidor.}


La lealtad del consumidor en un enfoque del comercio electrónico son las actitudes que toma el cliente según sus experiencias relacionadas a una marca o suministro, teniendo en cuenta las expectativas que le brindan en el momento dado de una empresa o entidad.

Según Barroso y Martín (1999) citado por Manzano, Pérez, Mafé, \& Blas (2010) nos menciona que la lealtad de los clientes al comercio electrónico tiende a crear ciertos constructos donde se ve la acogida de cuatro aspectos: el cliente está satisfecho con la compra y el servicio que le da dicha entidad donde participe, al ver la atención que le dan este repite la compra, que el cliente recomiende a la empresa a terceros y por último que reciba valor.

Por otro lado, Pàmies (2003) nos menciona tres tipos de lealtad del consumidor, la primera es la lealtad como comportamiento que se ve reflejado en las compras repetitivas de la misma marca o suministro, segundo es la lealtad como actitud que son incitadas por una fuerte disposición interna que tiene el cliente o consumidor, la tercera es la lealtad cognitiva que se rige por el compromiso del cliente o consumidor sin antes realizar un proceso de evaluación de sus causas que presenta en situaciones diferentes y ver cual le favorece más ante una situación de compra.

\subsubsection{Satisfacción del consumidor.}

La satisfacción al consumidor en un enfoque al comercio electrónico se define como las empresas brindan productos y/o servicios por medio de una web virtual cumpliendo y superando las expectativas del cliente y así satisfaciendo sus necesidades.

Currás \& Sánchez (2012), menciona que la satisfacción responde o tiende a cubrir las necesidades de los clientes o consumidores sea en el entorno tradicional o vía virtual. Por ello, se asume que los clientes que se sientan más satisfechos con una página Web brindaran una mayor predisposición a adquirir o seguir adquiriendo productos o servicios en la misma y así recomendarla a otras personas.

Oliver (1997) citado por Aldás, Currás, Ruiz, \& Sanz (2010), consideran que la satisfacción al consumidor como un juicio de naturaleza cognitivo-afectiva y relativa, es decir, es el resultado de la comparación entre una experiencia subjetiva y una base previa de referencia. No obstante, este autor propone que la satisfacción al consumidor es la valoración que se hace al consumidor, de una cualidad del producto o servicio proporciona o está proporcionando un resultado de agrado óptimo del consumo, incluyendo niveles por encima o por debajo de lo esperado.

\section{Conclusiones}


A través del estudio realizado mediante la revisión teórica de la variable comercio electrónico se llega a las siguientes conclusiones que recaban los aspectos más esenciales del desarrollo de este breve tratamiento teórico.

En el principio la expresión del comercio electrónico tuvo su aparición con la evolución de las actividades sencillas y es aquí donde su concepto se empieza a difundir en la comunidad académica y profesional. Por otro lado, a lo largo del tiempo se vinieron haciendo innovaciones desde las tareas más simples, hasta la llegada de la revolución industrial donde se ve el apogeo de la electricidad trayendo consigo la aparición de aparatos electrónicos, el internet con el comercio electrónico se desarrolló de formas similares en los años sesenta teniendo el comercio electrónico cuatro generaciones, en la primera se vio la importancia y el impacto, en la segunda vieron la oportunidad como estrategia, en la tercera se vio el marketing en la red y la implementación de bases de datos y por último en la cuarta se enfocaron en el cuidado del diseño y aspecto.

El modelo teórico propuesto por Fred D. Davis está especialmente diseñado para predecir la aceptación de los sistemas de información por los usuarios en las organizaciones, determinando el rechazo o aceptación de una determinada tecnología de información. En realidad, este modelo está relacionado con las convicciones, las actitudes, las intenciones y el comportamiento del usuario, para predecir la aceptación de una TI, sin embargo, el modelo de aceptación tecnológica en el enfoque del comercio electrónico es la dirección que tiene el usuario mediante su comportamiento, la voluntad que esta toma mediante su racionalidad ante una situación. Conforme a lo ilustrado el comercio electrónico es un nuevo método donde se realizan las transacciones compra y venta por internet tanto a nivel nacional e internacional, es una forma más rápida que te ayuda a generar valor para el cliente satisfaciendo sus necesidades.

La confianza del consumidor en un enfoque al comercio electrónico es dar seguridad al cliente a través de las plataformas brindadas por la empresa al momento de que este incluya sus datos.

La lealtad del consumidor se refiere cuando el cliente ha interactuado con la plataforma que ofrece la empresa y le muestra sus actitudes frente a sus experiencias que tuvo con la página web.

La Satisfacción del consumidor es cuando una empresa o entidad cubre esas necesidades que el cliente requiere a través de sus expectativas, dándole la valoración al cliente para que se sienta familiarizado con la empresa.

Es estos tiempos estamos viendo que el comercio electrónico está siendo percibida por las mismas personas que interactúan por un medio digital, de esa manera está creciendo 
por la misma necesidad de optimizar el tiempo y es más eficiente para tener una comunicación más rápida con los Stakeholders.

\section{Referencias}

Aldás, Currás, Ruiz., \& Sanz. (2010). Factores determinantes de la lealtad en el comercio electrónico B2C: aplicación a la compra de billetes de avión. Revista Española de Investigación de Marketing, 14(2), 113-142.

Aponte, J. (2013). Factores que influyen en la confianza del consumidor hacia el comercio electrónico: Un estudio en el área metropolitana de San Juan, Puerto Rico. Revista Empresarial Inter Metro, 9(2), 24-57.

Ballesteros, B., Tavera, J., \& Castaño, D. (2015). Aceptación tecnológica de la publicidad en dispositivos móviles en Colombia. Semestre Económico, 17 (36), 133-153. https://doi.org/10.22395/seec.v17n36a6

Bedregal, N., Tupacyupanqui, D., Cornejo, V., \& Flores, S. (2019). Evaluación de la percepción estudiantil en relación al uso de la plataforma Moodle desde la perspectiva del TAM, Ingeniare. Revista chilena de ingeniería, 27(4), 707-718. http://dx.doi.org/10.4067/S0718-33052019000400707

Ajzen, I. (2019). The Theory of Planned Behavior. Organizational Behavior and Human Decision Processes, 50(2), 179-211. https://doi.org/10.1016/0749-5978(91)90020-T

Belanger, F., Hiller, J. S., \& Smith, W. J. (2002). Trustworthiness in electronic commerce: the role of privacy, security, and site attributes. The journal of strategic Information Systems, 11(3-4), 245-270. https://doi.org/10.1016/S0963-8687(02)00018-5

Carola, J., Aderete, M., \& Motta, J. (2013). Adopción del comercio electrónico en Micro, Pequeñas y Medianas empresas comerciales y de servicios de Córdoba, Argentina. $\begin{array}{llll}\text { Cuadernos de } \quad \text { Administración, } & \text { 29(50). }\end{array}$ https://www.redalyc.org/articulo.oa?id=225029797006

Currás \& Sánchez (2012). Satisfacción y lealtad hacia un sitio Web: el efecto moderador del riesgo percibido. Esic Market, (141), 209-234. https://www.esic.edu/documentos/revistas/esicmk/120111_132708_E.pdf

Davis, F. (1989). Perceived Usefulness, Perceived Ease of Use, and User Acceptance of Information Technology. Management Information Systems Research Center, 13(3), 319-340. https://doi.org/10.2307/249008

Everett. (1995). Review Diffusion of Innovations, by Everett Rogers (1995). Stanford University, (1995), 5. https://doi.org/10.1525/aa.1963.65.5.02a00230 
Fransi, Ramón \& Fuentes (2013). El turismo y el comercio electrónico en España: un estudio del consumidor y de la empresa turística. Estudios Turísticos, (195), 79-99. https://dialnet.unirioja.es/servlet/articulo?codigo=4494054

Herrero, \& Rodríguez. (2001). La adopcion del comercio electronico B2C: Una comparacion empirica de los modelos alternativos, Revista Española de Investigación de Marketing ESIC, 10(1), 69-91. https://www.esic.edu/documentos/revistas/esicmk/070704_190628_E.pdf?id=26134

Jones, Alderete \& Motta (2014). Adopción del comercio electrónico en Micro, Pequeñas y Medianas empresas comerciales y de servici os de Córdoba, Argentina. Cuadernos de Administración, 29(50), 164-175. https://doi.org/10.25100/cdea.v29i50.52

Lee, \& Turban. (2001). A trust model for consumer internet shopping. International Journal of Electronic Commerce, 6(1), 75-91. https://doi.org/10.1080/10864415.2001.11044227

Malca, O. (2009). Comercio electrónico (1a ed. corregida). Lima: Universidad del Pacífico. Recuperado de http://hdl.handle.net/11354/76

Manzano, Pérez, Mafé, \& Blas. (2010). Factores determinantes de la lealtad en el comercio electrónico b2c. Aplicación a la compra de billetes de avión key drivers of loyalty in b2c e-commerce. Revista Española de Investigación de Marketing, 14(2), 113-142.

Myung, Chung \& Choong (2011). The effect of perceived trust on electronic commerce: Shopping online for tourism products and services in South Korea. Tourism Management, 32(2), 256-265. https://doi.org/10.1016/j.tourman.2010.01.011

Oropeza. (2018). El Comercio Electrónico y sus principios Económico-Comerciales. Retrieved from

www.juridicas.unam.mxhttps://biblio.juridicas.unam.mx/bjvLibrocompletoen:https://goo $. \mathrm{gl} / 4 \mathrm{P} 2 \mathrm{GYU}$

Robaina \& Melián (2006). La calidad percibida en el comercio electrónico B2C: el rol de las expectativas y las medidas de importancia. Revista Europea de Dirección y Economía de La Empresa, 15(1), 125-142.

Pàmies. (2003). La fidelidad del cliente en el ámbito de los servicios: un análisis de la escala "intenciones de comportamiento. Investigaciones Europeas de Dirección y Economía de La Empresa, 9(2), 189-204.

Ramírez-Correa, P.E., Rondán-Cataluña, F.J., \& Arenas-Gaitán, J.A. (2014). An empirical analysis of mobile Internet acceptance in Chile. Information Research, 19(3) http://InformationR.net/ir/19-3/paper635.html

Rios (2014). Análisis y perspectivas del comercio electrónico en México. Enl@ce: Revista Venezolana de Información, Tecnología y Conocimiento, 11(3), 97-121. https://www.redalyc.org/pdf/823/82332932006.pdf 
Rodríguez, Gutiérrez \& Herrero (2006). La adopción del comercio electrónico B2C: un a comparación empírica de dos modelos alternativos. Revista Española de Investigación de Marketing, 10(17), 69-92.

Romero, C., Alarcón, M., \& Gómez, M. (2011). Adopción de redes sociales virtuales: ampliación del modelo de aceptación tecnológica integrando confianza y riesgo percibido. Cuadernos de Economía y Dirección de la Empresa, 14(3), 194-205. https://doi.org/10.1016/j.cede.2010.12.003

Romero, \& Mauricio. (2012). Revisión de modelos de adopción de E-commerce para pymes de países en desarrollo. Revista de Investigación de Sistemas e Informática, RISI 9(1), 69-90.

Salazar \& Ramirez (2014). Efecto de los Talleres de Alfabetización Informacional en el uso de Bases de Datos Científicas. Formación universitaria, 7(3), 41-54. https://doi.org/10.4067/S0718-50062014000300006

Torres, \& Guerra. (2005). Comercio electrónico. Revista Internacional de Ciencias Sociales y Humanidades, SOCIOTAM, 79-116. Retrieved from http://www.profeco.gob.mx/internacionales/com_elec.asp

Tsuja \& Nishimura (2002). Adopcion e Implementacion del comercio electronico por empresas $\begin{array}{llll}\text { medianas en } & \text { Pl }\end{array}$ https://jefas.esan.edu.pe/index.php/jefas/article/view/131

Yong, L. A. (2004). Modelo de aceptación tecnológica. Revista Internacional de Ciencias Sociales y Humanidades, 1(1), 42. Retrieved from http://www.redalyc.org/pdf/654/65414107.pdf

Yong, L., Rivas, L., \& Chaparro, J. (2010). Modelo de aceptación tecnológica (tam): un estudio de la influencia de la cultura nacional y del perfil del usuario en el uso de las tic. INNOVAR. Revista de Ciencias Administrativas y Sociales, 20(36), 187-203. https://www.redalyc.org/articulo.oa?id=81819028014

Zolait, A. (2016). E-Mail as an Official Communication Tool in Bahrain: Individual and Public Organization Perspectives. International Journal of E-Services and Mobile Applications (IJESMA), 5(1), 51-76. https://doi.org/10.4018/jesma.2013010103 